\title{
REMARKS ON THE RAYLEIGH-BÉNARD CONVECTION ON SPHERICAL SHELLS
}

\author{
SHOUHONG WANG AND PING YANG
}

\begin{abstract}
The main objective of this article is to study the effect of spherical geometry on dynamic transitions and pattern formation for the Rayleigh-Bénard convection. The study is mainly motivated by the importance of spherical geometry and convection in geophysical flows. It is shown in particular that the system always undergoes a continuous (Type-I) transition to a $2 l_{c^{-}}$ dimensional sphere $S^{2 l_{c}}$, where $l_{c}$ is the critical wave length corresponding to the critical Rayleigh number. Furthermore, it has shown in [12] that it is critical to add nonisotropic turbulent friction terms in the momentum equation to capture the large-scale atmospheric and oceanic circulation patterns. We show in particular that the system with turbulent friction terms added undergoes the same type of dynamic transition, and obtain an explicit formula linking the critical wave number (pattern selection), the aspect ratio, and the ratio between the horizontal and vertical turbulent friction coefficients.
\end{abstract}

\section{CONTEnts}

1. Introduction 1

2. Boussinesq Equations 3

3. Linear Analysis and Principle of Exchange of Stability 5

4. Dynamical Transitions 8

5. Structure and Patterns of the Bénard Convection 9

6. Large-Scale Circulations and Turbulent Frictions 16

References 17

\section{INTRODUCTION}

The main objective of this article is to study the Bénard convection of fluids on a spherical shells. The main motivations are two-fold. First, convection plays an important role in the large scale atmospheric and oceanic circulations. The phenomena of the atmosphere and ocean are extremely rich in their organization and complexity, and involve a broad range of temporal and spatial scales [3]. To avoid to deal with the atmosphere and ocean in all their complexity, the main focus of this article is to study the Bénard convection on spherical shells to capture the main effects caused by the spherical geometry. Second, over the years, the Rayleigh-Bénard convection problem, together with the Taylor problem, has become one of the paradigms for studying nonequilibrium phase

Key words and phrases. Rayleigh-Bénard convection on spherical shells, dynamic transition, center manifold reduction, spherical harmonics, pattern formation.

The work was supported in part by the US Office of Naval Research and by the US National Science Foundation. 
transitions and pattern formation in nonlinear sciences; see among others Busse [1], Chandrasekhar [2], Cross [4], Ma and Wang [13], and the references therein.

For the Euclidean domain case, a systematic study on dynamic transition of the Rayleigh-Bénard convection is carried out in Ma and Wang [9, 11]. The main result in this direction is that the system always undergoes a Type-I (continuous) transition as the instability driving mechanism, namely Rayleigh number, crosses a critical threshold $R_{c}$, thanks to the symmetry of the linear operator, properties of the nonlinearity and asymptotic stability of the basic state at the critical threshold. Moreover, the system has a bifurcated attractor which is an $(m-1)$-dimensional homological sphere where $m$ is the number of critical eigenvalues of the linear operator.

In this article, we consider a fluid which occupies a layer of spherical shell modeled by the Boussinesq equations. Namely, the density function is treated as a constant except in the equation of state and in the buoyancy term. For simplicity, the spatial geometry of the domain is taken as the product of a two-dimensional sphere $S_{a}^{2}$ with radius $a$ and an interval: $S_{a}^{2} \times(0, h)$, where $h$ is the height of the fluid layer. This is mainly motivated by the fact that aspect ratio for the large scale atmosphere and ocean is small; see among others [7, 8, 14].

As in the Euclidean case, in the spherical shell case, we show that the system is always undergoes a Type-I (continuous) dynamic transition to a $2 l_{c}$-dimensional homological sphere $\Sigma_{R}$, where $l_{c}$ is the critical wave number determined by ${ }^{1}$

$$
\left[\frac{h}{a}\right]^{2} l_{c}\left(l_{c}+1\right)=\frac{\pi^{2}}{2} .
$$

An important part of this article is to show that $2 l_{c}$-dimensional homological sphere $\Sigma_{R}$ is in fact homeomorphic to a $2 l_{c}$-dimensional sphere $S^{2 l_{c}}$. This is achieved by carrying out the reduction of the original Boussinesq equations to the center manifold generated by the first $2 l_{c}+1$ modes. The main difficulty comes then from the nontrivial nonlinear interactions of the spherical harmonicsboth scalar and vectorial.

The remaining part of this article is devoted to the introduction of the turbulent friction terms in the Boussinesq equations. This is crucial for capturing the large scale convection patterns in the large scale geophysical flows, as demonstrated in thermohaline circulation studies by Ma and Wang [12]. The friction terms are added based on the convection scale considerations as introduced in [12], and are given by $\left(\sigma_{0} u, \sigma_{1} w\right)$; see (6.1). Under the assumption that

$$
1 \ll \sigma_{0} \ll \sigma_{1},
$$

the corresponding critical wave number $l_{c}$ is approximately given by

$$
\left[\frac{h}{a}\right]^{2} l_{c}\left(l_{c}+1\right) \sim \frac{\pi^{2}}{2}\left[\frac{\sigma_{0}}{\sigma_{1}}\right]^{1 / 2} .
$$

As we know, for the large-scale atmospheric and oceanic circulations, the critical wave number $l_{c}$ is often relatively small, e.g. $l_{c}=6$ for the global Walker circulation over the tropics. Hence the small aspect ratio $h / a$ is then balanced by the smallness of the ratio $\sigma_{0} / \sigma_{1}$ between the horizontal and vertical turbulent friction coefficients, leading to moderated size of the critical wave numbers.

It is worth mentioning that with the framework given in this article, one can study the humidity/salinity effects of the large scale atmospheric and oceanic flows, as well as the rotational effect (Coriolis force) of the earth. We shall report this elsewhere.

\footnotetext{
${ }^{1}$ The integer $l_{c}$ is to make the left-hand side the closet to the right-hand side.
} 
The article is organized as follows. Section 2 introduces the Boussinesq equations on the spherical shell, and the linear stability analysis is carried in Section 3. Sections 3 and 4 are on dynamic transitions and the structure of the bifurcated attractor. Section 6 demonstrates the need to introduction of convection scales, and studies the dynamics transitions for the Boussinesq equations with the added friction terms.

\section{Boussinesq Equations}

We start with the prototype of problem, the Bénard convection problem for a layer of fluid in a spherical shell. We consider the case where the fluid can be modeled by the Boussinesq equations. Namely, the density is considered as constant except in the buoyancy term and in the equation of state. The equations are given as follows:

$$
\begin{aligned}
& u_{t}+\left(u \cdot \nabla_{3}\right) u-\nu \Delta_{3} u+\rho_{0}^{-1} \nabla_{3} p=-g \vec{k}\left(1-\alpha\left(T-\overline{T_{0}}\right)\right), \\
& T_{t}+\left(u \cdot \nabla_{3}\right) T-\kappa \Delta_{3} T=0, \\
& \operatorname{div}_{3} u=0 .
\end{aligned}
$$

Here the unknown functions are velocity field $u=\left(u_{1}, u_{2}, u_{3}\right)$, the temperature function $T$, and the pressure function $p$. Other parameters include: $\nu$ is the kinematic viscosity, $\vec{k}=(0,0,1)$ is the unit vector in the vertical direction, $\mathrm{g}$ is the gravity constant, $\alpha$ is coefficient of thermal expansion of the fluid, $\overline{T_{0}}$ is temperature on the lower surface, $\rho_{0}$ is the density, $\kappa$ is he thermal diffusivity.For simplicity, we consider the spherical domain as

$$
\Omega_{d}=S_{a}^{2} \times(0, h),
$$

where $S_{a}^{2}$ is the two dimensional sphere with radius $\alpha$, and $h$ is the height of the fluid layer. The motion for this type of geometry is due to the study of large scale geophysical fluid flows, where the aspect ratio, and the thin layer of spherical shell can be approximated by $\Omega_{d}$ given here.

It's easy to get the basic solution:

$$
\begin{aligned}
& (u, T)=\left(0, \overline{T_{0}}-\beta x_{3}\right), \\
& p=p_{0}-\rho_{0} g\left(x_{3}+\frac{\alpha \beta}{2} x_{3}^{2}\right) .
\end{aligned}
$$

where

$$
\beta=\frac{\overline{T_{0}}-\overline{T_{1}}}{h}, r=\frac{a}{h}
$$

and $\overline{T_{1}}$ is temperature on the upper surface.

Mathematically speaking, it is convenient to introduce the nondimensional form of the equation. For this purpose, let

$$
\begin{aligned}
& (x, t)=\left(h x^{\prime}, \frac{h^{2} t^{\prime}}{\kappa}\right), \\
& (u, T)=\left(\frac{\kappa u,}{h}, \beta h \frac{T^{\prime}}{\sqrt{R}}+\overline{T_{0}}-\beta h x_{3}^{\prime}\right), \\
& p=\rho_{0} \frac{\kappa^{2} p^{\prime}}{h^{2}}+p_{0}-g \rho_{0}\left(h x_{3}^{\prime}+\frac{\alpha \beta h^{2}}{2}\left(x_{3}^{\prime}\right)^{2}\right),
\end{aligned}
$$

In addition, the Rayleigh number $R$ and the Prandtl number Pr are defined by

$$
R=\frac{g \alpha \beta}{\kappa \nu}, \operatorname{Pr}=\frac{\nu}{\kappa} .
$$


The nondimensional domain is

$$
\Omega=S_{r}^{2} \times(0,1)
$$

and it's reasonable to use the spherical coordinates. Let $(u, w)$ be the 3 dimensional velocity, $\mathrm{u}$ be the horizontal velocity field, and w be the vertical velocity. Also the following notations div, $\nabla$ are differential operators in the horizontal direction. Then the system (2.1) can be written as:

$$
\begin{aligned}
& \frac{1}{\operatorname{Pr}}\left(u_{t}+\nabla_{u} u+w \frac{\partial u}{\partial z}+\nabla p\right)-\left(\Delta+\frac{\partial^{2}}{\partial z^{2}}\right) u=0 \\
& \frac{1}{\operatorname{Pr}}\left(w_{t}+\nabla_{u} w+w \frac{\partial w}{\partial z}+\frac{\partial p}{\partial z}\right)-\left(\Delta+\frac{\partial^{2}}{\partial z^{2}}\right) w-\sqrt{R} T=0 \\
& T_{t}+\nabla_{u} T+w \frac{\partial w}{\partial z}-\sqrt{R} w-\left(\Delta+\frac{\partial^{2}}{\partial z^{2}}\right) T=0 \\
& \operatorname{div} u+\frac{\partial w}{\partial z}=0
\end{aligned}
$$

supplemented with the following free-slip boundary condition:

$$
w=0, T=0, \frac{\partial u}{\partial z}=0 \text { at } z=0,1
$$

It is worth mentioning that we can also study other physically sound boundary conditions such as the non-slip boundary or the periodic boundary conditions.

The following are some differential operators at spherical coordinates on $S_{r}^{2}$ :

$$
\begin{aligned}
& \nabla T=\frac{1}{r} \frac{\partial f}{\partial \theta} e_{\theta}+\frac{1}{r \sin \theta} \frac{\partial f}{\partial \varphi} e_{\varphi}, \\
& \operatorname{curl} T=\frac{1}{r \sin \theta} \frac{\partial f}{\partial \varphi} e_{\theta}-\frac{1}{r} \frac{\partial f}{\partial \theta} e_{\varphi}, \\
& \nabla_{u} v=\frac{1}{r}\left(u_{\theta} \frac{\partial v_{\theta}}{\partial \theta}+\frac{u_{\varphi}}{\sin \theta} \frac{\partial v_{\theta}}{\partial \varphi}-u_{\varphi} v_{\varphi} \cot \theta\right) e_{\theta}+\frac{1}{r}\left(u_{\theta} \frac{\partial v_{\varphi}}{\partial \theta}+\frac{u_{\varphi}}{\sin \theta} \frac{\partial v_{\varphi}}{\partial \varphi}+u_{\varphi} v_{\theta} \cot \theta\right) e_{\varphi}, \\
& \nabla_{u} T=\frac{1}{r}\left(u_{\theta} \frac{\partial T}{\partial \theta}+\frac{u_{\varphi}}{\sin \theta} \frac{\partial T}{\partial \varphi}\right), \\
& \Delta T=\frac{1}{r^{2}} \frac{1}{\sin \theta}\left[\frac{\partial}{\partial \theta}\left(\sin \theta \frac{\partial T}{\partial \theta}\right)+\frac{1}{\sin \theta} \frac{\partial^{2} T}{\partial \varphi^{2}}\right], \\
& \Delta u=\left(\Delta u_{\theta}-\frac{2 \cos \theta}{r^{2} \sin ^{2} \theta} \frac{\partial u_{\varphi}}{\partial \varphi}-\frac{u_{\theta}}{r^{2} \sin ^{2} \theta}\right) e_{\theta}+\left(\Delta u_{\varphi}+\frac{2 \cos \theta}{r^{2} \sin ^{2} \theta} \frac{\partial u_{\theta}}{\partial \varphi}-\frac{u_{\varphi}}{r^{2} \sin ^{2} \theta}\right) e_{\varphi} .
\end{aligned}
$$

where $u, v$ are vectors, and $T$ is scale.

For the problem (2.2) with (2.3), we define the function spaces as

$$
\begin{aligned}
& H=\left\{(u, w, T) \in L^{2}(\Omega)^{4} \mid \operatorname{div} u=0 ;(w, T)=0, \text { at } z=0,1\right\} \\
& H_{1}=\left\{(u, w, T) \in H^{2}(\Omega)^{4} \mid \operatorname{divu}=0 ;(w, T)=0, \frac{\partial u}{\partial z}=0 \text { at } z=0,1\right\} .
\end{aligned}
$$

Let $L_{\lambda}: H_{1} \rightarrow H$, and $G: H_{1} \rightarrow H$ be defined by 


$$
\begin{gathered}
L_{\lambda} \phi=P\left[\begin{array}{l}
\operatorname{Pr}\left(\Delta+\frac{\partial^{2}}{\partial z^{2}}\right) u \\
\operatorname{Pr}\left(\Delta+\frac{\partial^{2}}{\partial z^{2}}\right) w+\operatorname{Pr} \lambda T \\
\left(\Delta+\frac{\partial^{2}}{\partial z^{2}}\right) T+\lambda w
\end{array}\right], \\
G(\phi)=-P\left[\begin{array}{l}
\nabla_{u} u+w \frac{\partial u}{\partial z} \\
\nabla_{u} w+w \frac{\partial w}{\partial z} \\
\nabla_{u} T+w \frac{\partial T}{\partial z}
\end{array}\right],
\end{gathered}
$$

for any $\phi=(u, w, T) \in H_{1}$. Here $\lambda=\sqrt{R}$, and $P: L^{2}(\Omega)^{4} \rightarrow H$ is the Leray projection.

Then the equations (2.2) with (2.3) can be rewritten in the following operator form:

$$
\begin{array}{ll}
\frac{\partial \phi}{\partial t}=L_{\lambda} \phi+G(\phi), & \\
\phi=\phi_{0} & \text { for } t=0 .
\end{array}
$$

Here $\phi_{0} \in H$ is the initial condition. It is then classical to prove the existence and properties of solutions; see $[6,8]$; we omit the details here.

\section{Linear Analysis and Principle of Exchange of Stability}

To study the dynamic transitions of the Bénad convection problem (2.2) on the spherical shells, we need to consider the linear eigenvalue problem:

$$
L_{\lambda} \phi=\beta(\lambda) \phi,
$$

which is equivalent to

$$
\begin{aligned}
& \operatorname{Pr}\left(\Delta+\frac{\partial^{2}}{\partial z^{2}}\right) u-\nabla p=\beta(\lambda) u \\
& \operatorname{Pr}\left(\Delta+\frac{\partial^{2}}{\partial z^{2}}\right) w+\operatorname{Pr} \lambda T-\frac{\partial p}{\partial z}=\beta(\lambda) w \\
& \left(\Delta+\frac{\partial^{2}}{\partial z^{2}}\right) T+\lambda w=\beta(\lambda) T \\
& \operatorname{div}_{3}(u, w)=0
\end{aligned}
$$

supplemented with the boundary condition (2.3).

When $(u, w, T)$ doesn't depend on vertical $z$, then we have $(w, T)=0$, and $u=\operatorname{curl} Y_{l, m}$, so the eigenvalue and eigenfunctions are as follows:

$$
\begin{array}{rrr}
\beta_{l 0} & = & -\operatorname{Pr} \alpha_{l}^{2}, \\
\Psi_{l m 0} & = & \left(\operatorname{curl} Y_{l m}, 0,0\right) .
\end{array}
$$


Thanks to the spherical geometry of the domain, the above eigenvalue problem can solved using the following separation of variables:

$$
\begin{aligned}
& u=\nabla f(\theta, \varphi) H^{\prime}(z), \\
& w=\alpha^{2} f(\theta, \varphi) H(z), \\
& T=f(\theta, \varphi) \Theta(z) .
\end{aligned}
$$

By (3.4), we infer from (3.2) and (6.2) that

$$
\begin{aligned}
& -\Delta f=\alpha^{2} f, \\
& \operatorname{Pr}\left(-\alpha^{2} \nabla f H^{\prime}+\nabla f H^{\prime \prime \prime}\right)-\nabla p=\beta \nabla f H^{\prime}, \\
& \operatorname{Pr}\left(-\alpha^{4} f H+\alpha^{2} f H^{\prime \prime}+\lambda f \Theta\right)-\frac{\partial p}{\partial z}=\beta \alpha^{2} f H, \\
& -\alpha^{2} f \Theta+f \Theta^{\prime \prime}+\lambda \alpha^{2} f H=\beta f \Theta, \\
& \left(\Theta, H, H^{\prime \prime}\right)=0 \text { at } z=0,1 .
\end{aligned}
$$

For the eigenvalue problem (3.5) on the 2D sphere, the only possible candidates for the eigenfunction and eigenvalues are given by

$$
\begin{aligned}
& f=Y_{l m}(\theta, \varphi), \\
& \alpha_{l}^{2}=\frac{l(l+1)}{r^{2}} .
\end{aligned}
$$

To eliminate the pressure function $p$, we do $\frac{\partial(3.6)}{\partial z}-\nabla(3.7)$ :

$$
\Theta=\frac{\operatorname{Pr} H^{\prime \prime \prime \prime}-\left(2 \operatorname{Pr} \alpha^{2}+\beta\right) H^{\prime \prime}+\left(\alpha^{4} \operatorname{Pr}+\beta \alpha^{2}\right) H}{-\operatorname{Pr} \lambda} .
$$

Then we can consider two cases. First, for the case where $l=0$, it is easy to see that the eigenvalues and eigenvectors of $L_{\lambda}$ are:

$$
\begin{array}{rr}
\beta_{0 n} & =-n^{2} \pi^{2}, \\
\Psi_{00 n} & =(0,0, \sin n \pi z) .
\end{array}
$$

When $l \neq 0$, plug (3.12) into equation (3.8), then $H, \Theta$ are as follows:

$$
\begin{aligned}
& H_{n}=\sin n \pi z, \\
& \Theta_{n}=b_{l n} \sin n \pi z .
\end{aligned}
$$

Then plugging (3.14) and (3.15) into (3.12) and (3.8), we obtain that

$$
b_{l n}=\frac{\lambda \alpha_{l}^{2}}{\beta_{l n}+\gamma_{l n}^{2}}=\frac{\operatorname{Pr} \gamma_{l n}^{4}+\beta \gamma_{l n}^{2}}{-\operatorname{Pr} \lambda}
$$

where

$$
\gamma_{l n}^{2}=n^{2} \pi^{2}+\alpha_{l}^{2}=n^{2} \pi^{2}+\frac{l(l+1)}{r^{2}} .
$$

From (3.16), the eigenvalue $\beta$ solves the following quadratic equation:

$$
\beta^{2}+(1+\operatorname{Pr}) \gamma_{l n}^{2} \beta+\left(\gamma_{l n}^{4}-\frac{\lambda^{2} \alpha_{l}^{2}}{\gamma_{l n}^{2}}\right) \operatorname{Pr}=0
$$


we get two types of eigenvalues and eigenvectors:

$$
\begin{aligned}
& \beta_{l n}^{+}=\frac{1}{2}\left[-\gamma_{l n}^{2}(1+\operatorname{Pr})+\left(\gamma_{l n}^{4}(1+\operatorname{Pr})^{2}-4 \operatorname{Pr}\left(\gamma_{l n}^{4}-\frac{\lambda^{2} \alpha_{l}^{2}}{\gamma_{l n}^{2}}\right)\right)^{1 / 2}\right], \\
& \Psi_{l m n}^{+}=\left(n \pi \cos n \pi z \nabla Y_{l m}, \alpha_{l}^{2} Y_{l m} \sin n \pi z, \frac{\lambda \alpha_{l}^{2}}{\beta_{l n}^{+}+\gamma_{l n}^{2}} Y_{l m} \sin n \pi z\right), \\
& \beta_{l n}^{-}=\frac{1}{2}\left[-\gamma_{l n}^{2}(1+\operatorname{Pr})-\left(\gamma_{l n}^{4}(1+\operatorname{Pr})^{2}-4 \operatorname{Pr}\left(\gamma_{l n}^{4}-\frac{\lambda^{2} \alpha_{l}^{2}}{\gamma_{l n}^{2}}\right)\right)^{1 / 2}\right], \\
& \Psi_{l m n}^{-}=\left(n \pi \cos n \pi z \nabla Y_{l m}, \alpha_{l}^{2} Y_{l m} \sin n \pi z, \frac{\lambda \alpha_{l}^{2}}{\beta_{l n}^{-}+\gamma_{l n}^{2}} Y_{l m} \sin n \pi z\right) .
\end{aligned}
$$

So for problem (2.2) with boundary condition (2.3), the eigenfunctions for the corresponding linear operator as in (3.2) are consist of three group, which is shown in (3.3), (3.13), and (3.17).

Now we define the critical Rayleigh number $R_{c}=\lambda_{c}^{2}$ by

$$
\lambda_{c}=\min _{l, n} \frac{\left(n^{2} \pi^{2}+\alpha_{l}^{2}\right)^{3 / 2}}{\alpha_{l}}=\min _{l} \frac{\left(\pi^{2}+\alpha_{l}^{2}\right)^{3 / 2}}{\alpha_{l}} .
$$

It is easy to see that the minimum in the above formula is $\lambda_{c}$ is achieved at $\alpha_{l_{c}}^{2}$. By definition, we have (see (1.2)):

$$
\left[\frac{h}{a}\right]^{2} l_{c}\left(l_{c}+1\right)=\frac{\pi^{2}}{2} .
$$

As $l$ can only take integer values, it is possible for the minimum in (3.18) is achieved at two consecutive l's. For simplicity and without loss of generality, in this article, we only consider the case where the minimum is achieved at $\alpha_{l_{c}}$ with an integer $l_{c}$ :

$$
\lambda_{c}=\frac{\left(\pi^{2}+\alpha_{l_{c}}^{2}\right)^{3 / 2}}{\alpha_{l_{c}}}=\frac{3 \sqrt{3} \pi^{2}}{2} \quad \text { with } \quad \alpha_{l_{c}}=\frac{l_{c}\left(l_{c}+1\right)}{r^{2}},
$$

and the critical Rayleigh number is

$$
R_{c}=\lambda_{c}^{2}=\frac{27 \pi^{4}}{4}=657.5 .
$$

This value is consistent with the value developed classically; see among many others $[2,5]$.

By definition, the following principle of exchange of stability (PES) holds true:

$$
\begin{aligned}
& \beta_{l_{c} 1}^{+}(\lambda) \begin{cases}<0 & \text { if } \lambda<\lambda_{c}, \\
=0 & \text { if } \lambda<\lambda_{c}, \\
>0 & \text { if } \lambda>\lambda_{c},\end{cases} \\
& \beta_{l n}^{+}\left(\lambda_{c}\right)<0 \quad \forall(l, n) \neq\left(l_{c}, 1\right), l, n>0 \\
& \beta_{l n}^{-}\left(\lambda_{c}\right)<0 \quad \forall(l, n), l, n>0, \\
& \beta_{l 0}\left(\lambda_{c}\right)<0 \quad \forall l>0, \\
& \beta_{0 n}\left(\lambda_{c}\right)<0 \quad \forall n>0 .
\end{aligned}
$$

The critical eigenspace is then given by

$$
E_{1}=\left\{\sum_{m=-l_{c}}^{l_{c}} x_{m} \Psi_{l_{c} m 1}^{+}\left|x_{-m}=(-1)^{m} x_{m}^{*}, x_{m} \in \mathbb{C},\right| m \mid \leq l_{c}\right\}
$$


with dimension $\operatorname{dim} E_{1}=2 l_{c}+1$.

\section{Dynamical Transitions}

As mentioned in the introduction, the linear operator $L_{\lambda}$ is symmetric, leading to the real eigenvalues $\beta_{l, n}^{ \pm}, \beta_{0, n}$. With the PES at our disposal, we can obtain immediately that the Bénard convection system will undergoes a dynamic transition, and the following theorem shows that the transition is always Type-I.

Theorem 4.1. The Bénard convection problem (2.6) always undergoes a Type-I dynamic transition as the Rayleigh number $R$ crosses the critical value $R_{c}$. In particular, the following assertions hold true:

(1) When $R \leq R_{c}$, the basic motionless state with linear temperature profile $\phi=(u, w, T)=0$ is globally asymptotically stable.

(2) As $R>R_{c}$, but near $R_{c}$, the problem bifurcates to an attractor $\Sigma_{R}$, which is an $2 l_{c^{-}}$ dimensional homological sphere, where the integer $l_{c}$ is defined by (3.20).

(3) For any $\phi=(u, w, T) \in \Sigma_{R}$,

$$
\phi=\sum_{m=-1_{c}}^{l_{c}} x_{m} \Psi_{l_{c} m 1}^{+}+o\left(\sum_{m=-l_{c}}^{l_{c}}\left|x_{m}\right|\right), \quad x_{-m}=(-1)^{m} x_{m}^{*}, \quad x_{m} \in \mathbb{C},|m| \leq l_{c}
$$

(4) The attractor $\Sigma_{R}$ attracts $H \backslash \Gamma$, where $\Gamma$ is the stable manifold of $\phi=0$ with codimension $2 l_{c}+1$.

(5) The attractor $\Sigma_{R}$ contains at least one steady state solution of the problem.

Proof. As in [9], we can show that the basic motionless state is (globally) asymptotically stable the critical eigenvalue $\lambda=\lambda_{c}$. By the attractor bifurcation theorem in [10] and the linear analysis in the previous subsection, we obtain immediately Assertions (1)-(4) in the theorem.

It suffices then to show the existence of at least one steady state in the bifurcated attractor $\Sigma_{R}$. To this end, the steady state problem for (2.6) can be written as

$$
-A \phi-B_{\lambda} \phi=G(\phi)
$$

where $A+B_{\lambda}=L_{\lambda}$ and the linear operator $A$ and $B_{\lambda}$ are defined by

$$
A \phi=P\left[\begin{array}{c}
\operatorname{Pr}\left(\Delta+\frac{\partial^{2}}{\partial z^{2}}\right) u \\
\operatorname{Pr}\left(\Delta+\frac{\partial^{2}}{\partial z^{2}}\right) w \\
\left(\Delta+\frac{\partial^{2}}{\partial z^{2}}\right) T
\end{array}\right], \quad B_{\lambda} \phi=P\left[\begin{array}{l}
0 \\
\operatorname{Pr} \lambda T \\
\lambda w
\end{array}\right], \quad \forall \phi=(u, w, T) \in H_{1} .
$$

Obviously, the operator $A$ is invertible, and (4.1) can be written as

$$
\left[\mathrm{id}+(-A)^{-1} B_{\lambda}\right] \phi=(-A)^{-1} G(\phi) .
$$

Then it is classical to show that the linear operator id $+(-A)^{-1} B_{\lambda}: H \rightarrow H$ is a completely continuous field, and $(-A)^{-1} G: H \rightarrow H$ is a compact operator. Hence by the Krasnoselski bifurcation theorem (see e.g. Theorem 1.10 in [10]), (4.3) has a nontrivial steady state bifurcation at $R_{c}$, as the critical eigenvalue $R_{c}$ has odd multiplicity $2 l_{c}+1$, which is the dimension of the eigenspace $E_{1}$. The proof is complete. 


\section{Structure and Patterns of the Bénard Convection}

The above theorem shows that the system always undergoes a dynamic transition as $R$ crosses the critical Rayleigh number $R_{c}$, and the transition states occupy a set $\Sigma_{R}$, homological to $S^{2 l_{c}}$.

The main objective of this section is to derive more detailed structure of this bifurcated attractor. In particular, we shall give a strategy to show that $\Sigma_{R}$ is in fact homeomorphic to $S^{2 l_{c}}$.

The approach for this purpose is reduce the governing partial differential equations to the center manifold generated by the unstable modes for $R>R_{c}$ and near $R_{c}$. As the general case is tedious and time consuming, we present here only two special cases where critical wave number $l_{c}=1$ or 2. The other cases can be studied in the same fashion.

Theorem 5.1. Consider the Bénard convection in the case where $l_{c}=1$, which is equivalent in the dimensional form to

$$
\frac{h}{a}=\frac{\pi}{2}
$$

where $a$ is the horizontal length scale and $h$ is the vertical scale. Then the bifurcated attractor $\Sigma_{R}$ is homeomorphic to $S^{2}$, consisting of only degenerate steady states. Furthermore, $\Sigma_{R}$ is approximated by

$$
\Sigma_{R} \simeq\left\{\sum_{m=-1}^{1} \Psi_{1 m 1}^{+} \quad\left|x_{-m}=(-1)^{m} x_{m}^{*}, x_{0}\right|^{2}+\left|x_{1}\right|^{2}+\left|x_{-1}\right|^{2}=\beta_{11}^{+} / q_{1}\right\},
$$

where $q_{1}$ is given by

$$
q_{1}=\frac{3 \pi^{3}\left(17787+355912 \operatorname{Pr}-669713 \operatorname{Pr}^{2}+387787 \operatorname{Pr}^{3}\right)}{400000 \operatorname{Pr}\left(353-625 \operatorname{Pr}+353 \operatorname{Pr}^{2}\right)} .
$$

Namely, for any $\phi \in \Sigma_{R}$

$$
\begin{aligned}
& \phi=\sum_{m=-1}^{1} x_{m} \Psi_{1 m 1}^{+}+o\left(\left(\beta_{11}^{+} / q_{1}\right)^{1 / 2}\right), \\
& x_{0}^{2}+\left|x_{-1}\right|^{2}+\left|x_{1}\right|^{2}=\beta_{11}^{+} / q_{1}, \\
& x_{-m}=(-1)^{m} x_{m}^{*}, \quad|m| \leq 1 .
\end{aligned}
$$

Theorem 5.2. For the case where $l_{c}=2$, equivalently

$$
\frac{h}{a}=\frac{\pi}{2 \sqrt{3}}
$$

the bifurcated attractor $\Sigma_{R}$ is homeomorphic to $S^{4}$, which consists of at least an $S^{2}$ subset of degenerate steady states. Furthermore, when $\operatorname{Pr}=1, \Sigma_{R}$ is approximated by

$$
\Sigma_{R} \simeq\left\{\left.\sum_{m=-2}^{2} \Psi_{2 m 2}^{+} \quad\left|x_{-m}=(-1)^{m} x_{m}^{*}, \quad \sum_{m=-2}^{2}\right| x_{m}\right|^{2}=\beta_{21}^{+} / q_{2}\right\},
$$

where $q_{2}$ is given by

$$
q_{2}=\frac{2291405 \pi^{3}}{214754176}
$$

The proof of these two theorems is based on the reduction of the original Boussinesq equations to the center manifold generated by the first unstable modes, and to study the dynamics of the reduced system. As mentioned, the same method applies to other cases as well. 
Proof of Theorem 5.1. We proceed in several steps as follows.

Step 1. General Center Manifold Reduction Stratege. More precisely, let

$$
\begin{aligned}
& E_{1}=\left\{x=\sum_{m=-l_{c}}^{l_{c}} x_{m} \Psi_{l_{c} m 1}^{+}\left|x_{-m}=(-1)^{m} x_{m}^{*}, x_{m} \in \mathbb{C},\right| m \mid \leq l_{c}\right\}, \\
& E_{2}=E_{1}^{\perp} .
\end{aligned}
$$

The main idea of the center manifold function is to seek a function $\Phi$, which maps a neighborhood of the original in $E_{1}$ into $E_{2}$ given by

$$
\begin{aligned}
\Phi(x, \lambda)= & \sum_{l \geq 1,|m| \leq l} y_{l m 0} \Psi_{l m 0}+\sum_{n \geq 1} y_{00 n} \Psi_{00 n} \\
& +\sum_{(l, n) \neq l_{c}, 1, l, n>1} \sum_{|m| \leq l} y_{l m n}^{+} \Psi_{l m n}^{+}+\sum_{l, n>1} \sum_{|m| \leq l} y_{l m n}^{-} \Psi_{l m n}^{-} .
\end{aligned}
$$

By the approximation formula derived in [13], the center manifold function $\Phi$ can be approximated by

$$
-L_{\lambda} \Phi(x, \lambda)=P_{2} G(x)+o(2),
$$

where $P_{2}: H \rightarrow E_{2}$ is the canonical projection, and

$$
o(2)=o\left(\|x\|^{2}\right)+O\left(\left|\beta_{l_{c} 1}^{+}(\lambda)\right| \cdot\|x\|^{2}\right) .
$$

The first term in the right-hand side of (5.2) involves products of the eigenfunctions $\Psi_{l_{c} m 1}^{+}$, which are defined in terms of the spherical harmonics $Y_{l m}$ with $l \leq l_{c}$. Hence by the properties of the 3 -j symbols for the products of spherical harmonics, we only have to calculate the following center manifold function coefficients and all the others are zero or of higher-order:

(1) For $1 \leq l \leq 2 l_{c},|m| \leq l$,

$$
y_{l m 0}=-\frac{<G(x), \Psi_{l m 0}>}{\beta_{l 0}\left\|\Psi_{l m 0}\right\|^{2}}+o(2)
$$

(2) For $n=2$,

$$
y_{002}=-\frac{<G(x), \Psi_{002}>}{\beta_{02}\left\|\Psi_{002}\right\|^{2}}+o(2) ;
$$

(3) For $l \neq l_{c}, 1 \leq l \leq 2 l_{c},|m| \leq l$, and $n=0$ or 2 ,

$$
y_{l m n}^{+}=-\frac{<G(x), \Psi_{l m n}^{+}>}{\beta_{l n}^{+}\left\|\Psi_{l m n}^{+}\right\|^{2}}+o(2)
$$

(4) For $1 \leq l \leq 2 l_{c},|m| \leq l$, and $n=0$ or 2 ,

$$
y_{l m n}^{-}=-\frac{<G(x), \Psi_{l m n}^{-}>}{\beta_{l n}^{-}\left\|\Psi_{l m n}^{-}\right\|^{2}}+o(2)
$$

We note that hand-calculation of the right had sides of above formulas are still difficult if not impossible. Fortunately, one can write a simple mathematica program to perform symbolic calculations to derive precise formulas these coefficients.

Finally, the reduced equations are given as follows:

$$
\frac{d x_{m}}{d t}=\beta_{l_{c} 1}^{+}(\lambda) x_{m}+\frac{<G(X+\Phi(x, \lambda)), \Psi_{l_{c} m 1}^{+}>}{<\Psi_{l_{c} m 1}^{+}, \Psi_{l_{c} m 1}^{+}>} \quad \text { for }|m| \leq l_{c} .
$$


Again, to carry out the calculation for the nonlinear interaction in the right-hand side of this reduced equation, we need to use mathematica as well.

Step 2. Approximation of the Center manifold function for $l_{c}=1$. In this case, the first eigenvalue of (3.1) with (3.4) is

$$
\beta_{11}^{+}(\lambda)=\frac{1}{12}\left[-9 \pi^{2}(1+\operatorname{Pr})+\sqrt{81 \pi^{4}(-1+\operatorname{Pr})^{2}+48 \operatorname{Pr} \lambda^{2}}\right]
$$

Using Mathematica, we infer from (5.3)-(5.6) that:

$$
\begin{array}{ll}
y_{2-22}^{+}(x, \lambda)=\frac{1}{-\beta_{22}^{+}} d_{1}^{+} x_{-1}^{2}, & y_{2-22}^{-}(x, \lambda)=\frac{1}{-\beta_{22}^{-}} d_{1}^{-} x_{-1}^{2}, \\
y_{2-12}^{+}(x, \lambda)=\frac{1}{-\beta_{22}^{+}} \sqrt{2} d_{1}^{+} x_{-1} x_{0}, & y_{2-12}^{-}(x, \lambda)=\frac{1}{-\beta_{22}^{-}} \sqrt{2} d_{1}^{-} x_{-1} x_{0}, \\
y_{202}^{+}(x, \lambda)=\frac{1}{-\beta_{22}^{+}} \sqrt{\frac{3}{2}} d_{1}^{+}\left(x_{0}^{2}+x_{-1} x_{1}\right), & y_{202}^{-}(x, \lambda)=\frac{1}{-\beta_{22}^{-}} \sqrt{\frac{3}{2}} d_{1}^{-}\left(x_{0}^{2}+x_{-1} x_{1}\right), \\
y_{212}^{+}(x, \lambda)=\frac{1}{-\beta_{22}^{+}} \sqrt{2} d_{1}^{+} x_{0} x_{1}, & y_{212}^{-}(x, \lambda)=\frac{1}{-\beta_{22}^{-}} \sqrt{2} d_{1}^{-} x_{0} x_{1}, \\
y_{222}^{+}(x, \lambda)=\frac{1}{-\beta_{22}^{+}} d_{1}^{+} x_{1}^{2}, & y_{222}^{-}(x, \lambda)=\frac{1}{-\beta_{22}^{-}} d_{1}^{-} x_{1}^{2}, \\
y_{002}=-\frac{1}{64} \sqrt{3} \pi^{2}\left(x_{0}^{2}-2 x_{-1} x_{1}\right), & \\
y_{l m n}^{ \pm}=0, & \text { when }(l, n) \neq(2,2), l>0 .
\end{array}
$$

where

$$
\begin{aligned}
& d_{1}^{+}=3 \sqrt{\frac{3}{10}} \frac{\pi^{5 / 2}(121-121 \operatorname{Pr}+A)(187-121 \operatorname{Pr}+A)}{1936\left[-1493-1331 \operatorname{Pr}^{2}-11 A+\operatorname{Pr}(2500+11 A)\right]}, \\
& d_{1}^{-}=-3 \sqrt{\frac{3}{10}}\left(\pi^{5 / 2}\right) \frac{(-121+121 \operatorname{Pr}+A)(-187+121 \operatorname{Pr}+A)}{1936\left[1493+1331 \operatorname{Pr}^{2}-11 A+\operatorname{Pr}(-2500+11 A)\right]} \\
& \beta_{2,2}^{+}=\frac{1}{44} \pi^{2}(-121-121 \operatorname{Pr}+A), \\
& \beta_{2,2}^{-}=-\frac{1}{44} \pi^{2}(121+121 \mathrm{Pr}+A), \\
& A=\sqrt{11} \sqrt{1331-2338 \operatorname{Pr}+1331 \mathrm{Pr}^{2}} .
\end{aligned}
$$

Hence we obtain immediately the following second-order approximation of the center manifold function for $\lambda \geq \lambda_{c}$ :

$$
\begin{aligned}
\Phi(x, \lambda)= & y_{2-22}^{+} \Psi_{2-22}^{+}+y_{2-12}^{+} \Psi_{2-12}^{+}+y_{202}^{+} \Psi_{202}^{+} \\
& +y_{212}^{+} \Psi_{212}^{+}+y_{222}^{+} \Psi_{222}^{+} \\
& +y_{2-22}^{-} \Psi_{2-22}^{-}+y_{2-12}^{-} \Psi_{2-12}^{-}+y_{202}^{-} \Psi_{202}^{-} \\
& +y_{212}^{-} \Psi_{212}^{-}+y_{222}^{-} \Psi_{222}^{-}+y_{002} \Psi_{002}+o(2),
\end{aligned}
$$

with the coefficients given by (5.8). 
Step 3. Reduced equations. With the approximation of the center manifold function above, we deduce immediately following reduced equations:

$$
\begin{aligned}
& \frac{d x_{-1}}{d t}=\beta_{11}^{+} x_{-1}-q_{1} x_{-1}\left(x_{0}^{2}-2 x_{-1} x_{1}\right)+o(3) \\
& \frac{d x_{0}}{d t}=\beta_{11}^{+} x_{0}-q_{1} x_{0}\left(x_{0}^{2}-2 x_{-1} x_{1}\right)+o(3), \\
& \frac{d x_{1}}{d t}=\beta_{11}^{+} x_{1}-q_{1} x_{1}\left(x_{0}^{2}-2 x_{-1} x_{1}\right)+o(3),
\end{aligned}
$$

where

$$
\begin{aligned}
& q_{1}=\frac{3 \pi^{3}\left(17787+355912 \operatorname{Pr}-669713 \operatorname{Pr}^{2}+387787 \operatorname{Pr}^{3}\right)}{400000 \operatorname{Pr}\left(353-625 \operatorname{Pr}+353 \operatorname{Pr}^{2}\right)}, \\
& o(3)=o\left(\|x\|^{3}\right)+O\left(\left|\beta_{l_{c} 1}^{+}\right| \cdot\|x\|^{3}\right) .
\end{aligned}
$$

As the eigenfunction are complex, we can set

$$
x_{m}=\frac{1}{\sqrt{2}}\left(y_{m}+i z_{m}\right), \quad y_{m}, z_{m} \in \mathbb{R} .
$$

By definition (see (3.22)), we have

$$
y_{m}=(-1)^{m} y_{-m}, z_{m}=(-1)^{m+1} z_{-m}, m>0 .
$$

Hence we obtain the following reduced equations:

$$
\begin{aligned}
& \frac{d y_{1}}{d t}=\beta_{11}^{+} y_{1}-q_{1} y_{1}\left(x_{0}^{2}+y_{1}^{2}+z_{1}^{2}\right)+o(3), \\
& \frac{d x_{0}}{d t}=\beta_{11}^{+} x_{0}-q_{1} x_{0}\left(x_{0}^{2}+y_{1}^{2}+z_{1}^{2}\right)+o(3), \\
& \frac{d z_{1}}{d t}=\beta_{11}^{+} z_{1}-q_{1} z_{1}\left(x_{0}^{2}+y_{1}^{2}+z_{1}^{2}\right)+o(3) .
\end{aligned}
$$

STEP 4. Let $g$ be the cubic terms in the right hand side of the reduced equation. Then, we have

$$
-\left(g,\left(y_{1}, x_{0}, z_{1}\right)\right) \leq q_{1}\left(x_{0}^{2}+y_{1}^{2}+z_{1}^{2}\right)^{2} .
$$

Hence by the attractor bifurcation theorem in [13], the bifurcated attractor $\Sigma_{R}$ is homeomorphic to $S^{2}$.

Also, by Theorem 4.1, there is at least one steady state solution in $\Sigma_{R}$. In addition, due to the spherical geometry, the original Boussinesq equations have an $S^{2}$-symmetry. Hence, this steady state solution generates an $S^{2}$-set of steady states. Therefore, $\Sigma_{R}$ consists precisely steady state solutions.

Finally, by setting the right hand-side of the above reduced equations (5.11) equal to zero, we obtain that $\Sigma_{R}$ is approximated by

$$
\left\{\left(y_{1}, x_{0}, z_{1}\right) \in \mathbb{R}^{3} \quad \mid \quad x_{0}^{2}+y_{1}^{2}+z_{1}^{2}=\beta_{11}^{+} / q_{1}\right\} .
$$

The proof is complete.

Proof of Theorem 5.2. We note that the unstable space $E_{1}$ is now given by

$$
E_{1}=\left\{x=\sum_{m=-2}^{2} x_{m} \Psi_{2 m 1}^{+}\left|x_{-m}=(-1)^{m} x_{m}^{*}, x_{m} \in \mathbb{C},\right| m \mid \leq l_{c}\right\},
$$


and we still use for brevity $x$ to denote

$$
x=\sum_{m=-2}^{2} x_{m} \Psi_{2 m 1}^{+}
$$

As in the previous proof, the approximation of the center manifold function is then given by

$$
\begin{aligned}
\Phi(x, \lambda)= & y_{2-22}^{+} \Psi_{2-22}^{+}+y_{2-12}^{+} \Psi_{2-12}^{+}+y_{202}^{+} \Psi_{202}^{+}+y_{212}^{+} \Psi_{212}^{+}+y_{222}^{+} \Psi_{222}^{+} \\
& +y_{4-42}^{+} \Psi_{4-42}^{+}+y_{4-32}^{+} \Psi_{4-32}^{+}+y_{4,-2,2}^{+} \Psi_{4-22}^{+}+y_{4-12}^{+} \Psi_{4-12}^{+}+y_{402}^{+} \Psi_{402}^{+} \\
& +y_{412}^{+} \Psi_{412}^{+}+y_{422}^{+} \Psi_{422}^{+}+y_{4,3,2}^{+} \Psi_{432}^{+}+y_{442}^{+} \Psi_{442}^{+} \\
& +y_{2-22}^{-} \Psi_{2-22}^{-}+y_{2-12}^{-} \Psi_{2-12}^{-}+y_{2,0,2}^{-} \Psi_{202}^{-}+y_{212}^{-} \Psi_{212}^{-}+y_{222}^{-} \Psi_{222}^{-} \\
& +y_{4-42}^{-} \Psi_{4-42}^{-}+y_{4-32}^{-} \Psi_{4-32}^{-}+y_{4-22}^{-} \Psi_{4-22}^{-}+y_{4-12}^{-} \Psi_{4-12}^{-}+y_{402}^{-} \Psi_{402}^{-} \\
& +y_{412}^{-} \Psi_{412}^{-}+y_{422}^{-} \Psi_{422}^{-}+y_{432}^{-} \Psi_{432}^{-}+y_{442}^{-} \Psi_{442}^{-}+y_{002} \Psi_{002}+o(2)
\end{aligned}
$$


where the non vanishing coefficients in the above center manifold function are as follows:

$$
\begin{aligned}
& y_{2-22}^{+}\left(x, \lambda_{c}\right)=\frac{c_{1}^{+}\left(\sqrt{6} x_{-1}^{2}-4 x_{-2} x_{0}\right)}{-\beta_{22}^{+}}, \\
& y_{2-22}^{-}\left(x, \lambda_{c}\right)=\frac{c_{1}^{-}\left(\sqrt{6} x_{-1}^{2}-4 x_{-2} x_{0}\right.}{-\beta_{22}^{-}}, \\
& y_{2-12}^{+}\left(x, \lambda_{c}\right)=\frac{2 c_{1}^{+}\left(x_{-1} x_{0}-\sqrt{6} x_{-2} x_{1}\right)}{-\beta_{22}^{+}}, \\
& y_{2-12}^{-}\left(x, \lambda_{c}\right)=\frac{2 c_{1}^{-}\left(x_{-1} x_{0}-\sqrt{6} x_{-2} x_{1}\right)}{-\beta_{22}^{-}}, \\
& y_{202}^{+}\left(x, \lambda_{c}\right)=\frac{2 c_{1}^{+}\left(x_{0}^{2}-x_{-1} x_{1}-2 x_{-2} x_{2}\right)}{-\beta_{22}^{+}}, \\
& y_{202}^{-}\left(x, \lambda_{c}\right)=\frac{2 c_{1}^{-}\left(x_{0}^{2}-x_{-1} x_{1}-2 x_{-2} x_{2}\right)}{-\beta_{22}^{-}}, \\
& y_{212}^{+}\left(x, \lambda_{c}\right)=\frac{2 c_{1}^{+}\left(x_{0} x_{1}-\sqrt{6} x_{-1} x_{2}\right)}{-\beta_{22}^{+}}, \\
& y_{212}^{-}\left(x, \lambda_{c}\right)=\frac{2 c_{1}^{-}\left(x_{0} x_{1}-\sqrt{6} x_{-1} x_{2}\right)}{-\beta_{22}^{-}}, \\
& y_{222}^{+}\left(x, \lambda_{c}\right)=\frac{c_{1}^{+}\left(\sqrt{6} x_{1}^{2}-4 x_{0} x_{2}\right)}{-\beta_{22}^{+}}, \\
& y_{222}^{-}\left(x, \lambda_{c}\right)=\frac{c_{1}^{-}\left(\sqrt{6} x_{1}^{2}-4 x_{0} x_{2}\right)}{-\beta_{22}^{-}}, \\
& y_{4-42}^{+}\left(x, \lambda_{c}\right)=\frac{c_{2}^{+}}{-\beta_{42}^{+}} x_{-2}^{2}, \\
& y_{4-42}^{-}\left(x, \lambda_{c}\right)=\frac{c_{2}^{-}}{-\beta_{42}^{-}} x_{-2}^{2}, \\
& y_{4-32}^{+}\left(x, \lambda_{c}\right)=\frac{\sqrt{2} c_{2}^{+}}{-\beta_{42}^{+}} x_{-2} x_{-1}, \\
& y_{4-32}^{-}\left(x, \lambda_{c}\right)=\frac{\sqrt{2} c_{2}^{-}}{-\beta_{42}^{-}} x_{-2} x_{-1}, \\
& y_{4-22}^{+}\left(x, \lambda_{c}\right)=\frac{\sqrt{5} c_{3}^{+}}{-\beta_{42}^{+}}\left(\sqrt{2} x_{-1}^{2}+\sqrt{3} x_{-2} x_{0}\right), \\
& y_{4-22}^{-}\left(x, \lambda_{c}\right)=\frac{\sqrt{5} c_{3}^{-}}{-\beta_{42}^{-}}\left(\sqrt{2} x_{-1}^{2}+\sqrt{3} x_{-2} x_{0}\right), \\
& y_{4-12}^{+}\left(x, \lambda_{c}\right)=\frac{\sqrt{5} c_{3}^{+}}{-\beta_{42}^{+}}\left(\sqrt{6} x_{-1} x_{0}+x_{-2} x_{1}\right), \\
& y_{4-12}^{-}\left(x, \lambda_{c}\right)=\frac{\sqrt{5} c_{3}^{-}}{-\beta_{42}^{-}}\left(\sqrt{6} x_{-1} x_{0}+x_{-2} x_{1}\right), \\
& y_{402}^{+}\left(x, \lambda_{c}\right)=\frac{c_{3}^{+}}{-\beta_{42}^{+}}\left(3 x_{0}^{2}+4 x_{-1} x_{1}+x_{-2} x_{2}\right), \\
& y_{402}^{-}\left(x, \lambda_{c}\right)=\frac{c_{3}^{-}}{-\beta_{42}^{-}}\left(3 x_{0}^{2}+4 x_{-1} x_{1}+x_{-2} x_{2}\right) \text {, } \\
& y_{412}^{+}\left(x, \lambda_{c}\right)=\frac{\sqrt{5} c_{3}^{+}}{-\beta_{42}^{+}}\left(\sqrt{6} x_{0} x_{1}+x_{-1} x_{2}\right), \\
& y_{412}^{-}\left(x, \lambda_{c}\right)=\frac{\sqrt{5} c_{3}^{-}}{-\beta_{42}^{-}}\left(\sqrt{6} x_{0} x_{1}+x_{-1} x_{2}\right), \\
& y_{422}^{+}\left(x, \lambda_{c}\right)=\frac{\sqrt{5} c_{3}^{+}}{-\beta_{42}^{+}}\left(\sqrt{2} x_{1}^{2}+\sqrt{3} x_{0} x_{2}\right), \\
& y_{422}^{-}\left(x, \lambda_{c}\right)=\frac{\sqrt{5} c_{3}^{-}}{-\beta_{42}^{-}}\left(\sqrt{2} x_{1}^{2}+\sqrt{3} x_{0} x_{2}\right), \\
& y_{432}^{+}\left(x, \lambda_{c}\right)=\frac{\sqrt{2} c_{2}^{+}}{-\beta_{42}^{+}} x_{1} x_{2}, \\
& y_{432}^{-}\left(x, \lambda_{c}\right)=\frac{\sqrt{2} c_{2}^{-}}{-\beta_{42}^{-}} x_{1} x_{2}, \\
& y_{442}^{+}\left(x, \lambda_{c}\right)=\frac{c_{2}^{+}}{-\beta_{42}^{+}} x_{2}^{2}, \\
& y_{442}^{-}\left(x, \lambda_{c}\right)=\frac{c_{2}^{-}}{-\beta_{42}^{-}} x_{2}^{2}, \\
& y_{002}=-\frac{1}{16} \sqrt{3} \pi^{4}\left(x_{0}^{2}-2 x_{-1} x_{1}+2 x_{-2} x_{2}\right),
\end{aligned}
$$


where

$$
\begin{aligned}
& c_{1}^{+}=\frac{\sqrt{5} \pi^{5 / 2}(9-9 \operatorname{Pr}+B)(15-9 \operatorname{Pr}+B)}{672\left[-29-27 \operatorname{Pr}^{2}-3 B+\operatorname{Pr}(52+3 B)\right]}, \\
& c_{2}^{+}=\frac{3 \sqrt{\frac{5}{14}} \pi^{5 / 2}\left(-1-\frac{153}{(289-289 \operatorname{Pr}+\sqrt{17} C)}\right)}{136\left(1+\frac{20655}{(289-289 \operatorname{Pr}+\sqrt{17} C)^{2}}\right)}, \\
& c_{3}^{+}=\frac{3 \pi^{5 / 2}(442-289 \operatorname{Pr}+\sqrt{17} C)(289-289 \operatorname{Pr}+\sqrt{17} C)}{16184\left(-11041-9826 \operatorname{Pr}^{2}-34 \sqrt{17} C+\operatorname{Pr}(18437+34 \sqrt{17} C)\right)}, \\
& c_{1}^{-}=-\frac{\sqrt{5} \pi^{5 / 2}(-9+9 \operatorname{Pr}+B)(-15+9 \operatorname{Pr}+B)}{672\left(29+27 \operatorname{Pr}^{2}-3 B+\operatorname{Pr}(-52+3 B)\right)}, \\
& c_{2}^{-}=\frac{3 \sqrt{\frac{5}{14}} \pi^{5 / 2}\left(-1+\frac{153}{(-289+289 \operatorname{Pr}+\sqrt{17} C)}\right)}{136\left(1+\frac{20655}{(-289+289 \operatorname{Pr}+\sqrt{17} C)^{2}}\right)}, \\
& c_{3}^{-}=\frac{-3 \pi^{5 / 2}(-442+289 \operatorname{Pr}+\sqrt{17} C)(-289+289 \operatorname{Pr}+\sqrt{17} C)}{16184\left(11041+9826 \operatorname{Pr}{ }^{2}-34 \sqrt{17} C+\operatorname{Pr}(-18437+34 \sqrt{17} C)\right)} .
\end{aligned}
$$

Here

$$
\begin{aligned}
& B=\sqrt{81-150 \mathrm{Pr}+81 \mathrm{Pr}^{2}}, \\
& C=\sqrt{4913-8611 \mathrm{Pr}+4913 \mathrm{Pr}^{2}},
\end{aligned}
$$

and $\beta_{2,2}^{+}, \beta_{4,2}^{+}, \beta_{2,2}^{-}, \beta_{4,2}^{-}$are given by $(3.17)$.

For simplicity, we only consider the case where $\operatorname{Pr}=1$. Then the reduced equation are as following:

$$
\begin{aligned}
\frac{d x_{-2}}{d t} & =\beta_{21}^{+} x_{-2}-q_{2} x_{-2}\left(x_{0}^{2}-2 x_{-1} x_{1}+2 x_{-2} x_{2}\right)+o(3), \\
\frac{d x_{-1}}{d t} & =\beta_{21}^{+} x_{-1}-q_{2} x_{-1}\left(x_{0}^{2}-2 x_{-1} x_{1}+2 x_{-2} x_{2}\right)+o(3), \\
\frac{d x_{0}}{d t} & =\beta_{21}^{+} x_{0}-q_{2} x_{0}\left(x_{0}^{2}-2 x_{-1} x_{1}+2 x_{-2} x_{2}\right)+o(3), \\
\frac{d x_{1}}{d t} & =\beta_{21}^{+} x_{1}-q_{2} x_{1}\left(x_{0}^{2}-2 x_{-1} x_{1}+2 x_{-2} x_{2}\right)+o(3), \\
\frac{d x_{2}}{d t} & =\beta_{21}^{+} x_{2}-q_{2} x_{2}\left(x_{0}^{2}-2 x_{-1} x_{1}+2 x_{-2} x_{2}\right)+o(3),
\end{aligned}
$$

where

$$
q_{2}=\frac{2291405 \pi^{3}}{214754176}
$$

Let

$$
x_{m}=\frac{1}{\sqrt{2}}\left(y_{m}+i z_{m}\right), \quad y_{m}, z_{m} \in \mathbb{R}
$$

Then

$$
y_{m}=(-1)^{m} y_{-m}, \quad z_{m}=(-1)^{m+1} z_{-m}, m>0 .
$$


We derive then the reduced equations as follows:

$$
\begin{aligned}
\frac{d y_{1}}{d t} & =\beta_{21}^{+} y_{1}-q_{2} y_{1}\left(x_{0}^{2}+y_{1}^{2}+z_{1}^{2}+y_{2}^{2}+z_{2}^{2}\right)+o(3), \\
\frac{d y_{2}}{d t} & =\beta_{21}^{+} y_{2}-q_{2} y_{2}\left(x_{0}^{2}+y_{1}^{2}+z_{1}^{2}+y_{2}^{2}+z_{2}^{2}\right)+o(3), \\
\frac{d x_{0}}{d t} & =\beta_{21}^{+} x_{0}-q_{2} x_{0}\left(x_{0}^{2}+y_{1}^{2}+z_{1}^{2}+y_{2}^{2}+z_{2}^{2}\right)+o(3), \\
\frac{d z_{1}}{d t} & =\beta_{21}^{+} z_{1}-q_{2} z_{1}\left(x_{0}^{2}+y_{1}^{2}+z_{1}^{2}+y_{2}^{2}+z_{2}^{2}\right)+o(3), \\
\frac{d z_{2}}{d t} & =\beta_{21}^{+} z_{2}-q_{2} z_{2}\left(x_{0}^{2}+y_{1}^{2}+z_{1}^{2}+y_{2}^{2}+z_{2}^{2}\right)+o(3) .
\end{aligned}
$$

Then as in the proof of last theorem, we can show that $\Sigma_{R}$ is homeomorphic to $S^{4}$, and we have the following approximation:

$$
\Sigma_{R} \simeq\left\{\left(y_{2}, y_{1}, x_{0}, z_{1}, z_{2}\right) \in \mathbb{R}^{5} \quad \mid \quad x_{0}^{2}+y_{1}^{2}+z_{1}^{2}+y_{2}^{2}+z_{2}^{2}=\beta_{21}^{+} / q_{2}\right\},
$$

and $\Sigma_{R}$ contains at least an $S^{2}$ subset of steady state solutions. We remark here that if we retain only the linear and cubic order terms in the above reduced equations (5.15), we would have the whole $S^{4}$ consisting only steady state solutions. However, since the steady states solutions are degenerate (with zero Jacobian), there is no persistence and stability of these steady state solutions.

The proof of the theorem is complete.

\section{Large-Scale Circulations and Turbulent Frictions}

Now we consider the following equations:

$$
\begin{aligned}
& \frac{1}{\operatorname{Pr}}\left(u_{t}+\nabla_{u} u+w \frac{\partial u}{\partial z}+\nabla p\right)+\sigma_{0} u-\left(\Delta+\frac{\partial^{2}}{\partial z^{2}}\right) u=0, \\
& \frac{1}{\operatorname{Pr}}\left(w_{t}+\nabla_{u} w+w \frac{\partial w}{\partial z}+\frac{\partial p}{\partial z}\right)+\sigma_{1} w-\left(\Delta+\frac{\partial^{2}}{\partial z^{2}}\right) w-\sqrt{R} T=0, \\
& T_{t}+\nabla_{u} T+w \frac{\partial w}{\partial z}-\sqrt{R} w-\left(\Delta+\frac{\partial^{2}}{\partial z^{2}}\right) T=0, \\
& \operatorname{div} u+\frac{\partial w}{\partial z}=0,
\end{aligned}
$$

supplemented with boundary condition:

$$
w=0, T=0, \frac{\partial u}{\partial z}=0 \text { at } z=0,1
$$

By similar computation, we get the three groups of eigenvalues and eigenvectors:

(1) For $(w, T)=0$

$$
\begin{aligned}
& \beta_{l 0}=-\operatorname{Pr}\left(\alpha_{l}^{2}+\sigma_{0}\right), \\
& \Psi_{i m 0}=\left(\operatorname{curl} Y_{l m}, 0,0\right) ;
\end{aligned}
$$

(2) For $l=0$ with $n \geq 1$

$$
\begin{aligned}
& \beta_{0 n}=-n^{2} \pi^{2}, \\
& \Psi_{00 n}=(0,0, \sin n \pi z) ;
\end{aligned}
$$


(3) For $l, n \geq 1$

$$
\begin{aligned}
& \beta_{l n}^{+}=-\frac{1}{2} D+\frac{1}{2} \sqrt{D^{2}-4 \operatorname{Pr} E}, \\
& \Psi_{l m n}^{+}=\left(n \pi \cos n \pi z \nabla Y_{l m}, \alpha_{l}^{2} Y_{l m} \sin n \pi z, \frac{2 \lambda \alpha_{l}^{2}}{\beta_{l n}^{+}+\gamma_{l n}^{2}} Y_{l m} \sin n \pi z\right), \\
& \beta_{l n}^{-}=-\frac{1}{2} D-\frac{1}{2} \sqrt{D^{2}-4 \operatorname{Pr} E}, \\
& \Psi_{l m n}^{-}=\left(n \pi \cos n \pi z \nabla Y_{l m}, \alpha_{l}^{2} Y_{l m} \sin n \pi z, \frac{2 \lambda \alpha_{l}^{2}}{\beta_{l n}^{-}+\gamma_{l n}^{2}} Y_{l, m} \sin n \pi z\right) .
\end{aligned}
$$

Here

$$
\begin{aligned}
& D=\gamma_{l n}^{2}(1+\operatorname{Pr})+\frac{\operatorname{Pr}\left(\sigma_{1} \alpha_{l}^{2}+\sigma_{0} n^{2} \pi^{2}\right)}{\gamma_{l n}^{2}}, \\
& E=\left(\gamma_{l n}^{4}-\frac{\lambda^{2} \alpha_{l}^{2}}{\gamma_{l n}^{2}}+\sigma_{1} \alpha_{l}^{2}+\sigma_{0} n^{2} \pi^{2}\right) .
\end{aligned}
$$

To get the critical Rayleigh number $\lambda_{c}$, let $\beta_{l, n}=0$, and obtain that

$$
\lambda_{c}=\min _{l, n>0} \frac{\gamma_{l n}}{\alpha_{l}} \sqrt{\left(\gamma_{l n}^{4}+\sigma_{1} \alpha_{l}^{2}+\sigma_{0} n^{2} \pi^{2}\right)}=\min _{l>0} \sqrt{\frac{\left.\left(\alpha_{l}^{2}+\pi^{2}\right)\left[\left(\alpha_{l}^{1}+\pi^{2}\right)^{2}+\sigma_{1} \alpha_{l}^{2}+\sigma_{0} \pi^{2}\right)\right]}{\alpha_{l}^{2}}} .
$$

By the analysis for the convection scales in [13], we consider the case where the turbulent friction coefficients satisfy

$$
1 \ll \sigma_{0} \ll \sigma_{1} .
$$

In this case, the minimum in (6.3) is approximately achieved at

$$
\left[\frac{h}{a}\right]^{2} l_{c}\left(l_{c}+1\right)=\alpha_{l}^{2} \sim \frac{\pi^{2}}{2}\left[\frac{\sigma_{0}}{\sigma_{1}}\right]^{1 / 2} .
$$

Then the same results as Theorems 4.1, 5.1 and 5.2 hold true for the Rayleigh-Bénard convection system (6.1) with added turbulent friction terms. We omit the details. It is worth, however, to remark that (6.5) provides a precise pattern selection mechanism in terms of the aspect ratio $h / a$, and the ratio between the horizontal and vertical friction coefficients. For example, for the circulation, we can choose the parameters as follows:

$$
a=6.4 \times 10^{6} \mathrm{~m}, \quad h=10^{4} \mathrm{~m}, \quad l_{c}=6
$$

Then,

$$
\sigma_{0} / \sigma_{1} \approx 10^{-8} .
$$

Here $l_{c}=6$ represents the 6 Walker circulation cells over the tropical, and is also consistent with the three meridional circulation cells-the Hadley cell, the midlatitude cell and the polar cell, in the Northern hemisphere.

\section{REFERENCES}

[1] F. Busse, Non-linear properties of thermal convection, Reports on Progress in Physics, 41 (1978), p. 1929.

[2] S. Chandrasekhar, Hydrodynamic and Hydromagnetic Stability, Dover Publications, Inc., 1981.

[3] J. Charney, On the scale of atmospheric motion, Geofys. Publ., 17(2) (1948), pp. 1-17.

[4] M. Cross and P. Hohenberg, Pattern formation outside of equilibrium, Reviews of Modern Physics, (1993).

[5] P. Drazin and W. Reid, Hydrodynamic Stability, Cambridge University Press, 1981. 
[6] C. Foins, O. Manley, and R. Temam, Attractors for the Bénard problem: existence and physical bounds on their fractal dimension, Nonlinear Anal., 11 (1987), pp. 939-967.

[7] J.-L. Lions, R. Temam, and S. Wang, New formulations of the primitive equations of atmosphere and applications, Nonlinearity, 5 (1992), pp. 237-288.

[8] - On the equations of the large-scale ocean, Nonlinearity, 5 (1992), pp. 1007-1053.

[9] T. MA And S. Wang, Dynamic bifurcation and stability in the Rayleigh-Bénard convection, Commun. Math. Sci., 2 (2004), pp. 159-183.

[10] — Bifurcation theory and applications, vol. 53 of World Scientific Series on Nonlinear Science. Series A: Monographs and Treatises, World Scientific Publishing Co. Pte. Ltd., Hackensack, NJ, 2005.

[11] — Rayleigh-Bénard convection: dynamics and structure in the physical space, Commun. Math. Sci., 5 (2007), pp. 553-574.

[12] - Dynamic transition theory for thermohaline circulation, Physica D, 239:3-4 (2010), pp. 167-189.

[13] - Phase Transition Dynamics in Nonlinear Sciences, submitted, 2011.

[14] J. Pedlosky, Geophysical Fluid Dynamics, Springer-Verlag, New-York, second ed., 1987.

(Wang) Department of Mathematics, Indiana University, Bloomington, IN 47405

E-mail address: showang@indiana.edu, http://www.indiana.edu/ fluid

(Yang) Department of Mathematics, Indiana University, Bloomington, IN 47405

E-mail address: pingyang@umail.iu.edu 